University of South Carolina

Scholar Commons

4-8-1985

\title{
Origin of the Peaked Structure in the Conductance of One- Dimensional Silicon Accumulation Layers
}

Richard A. Webb

University of South Carolina - Columbia, webbra@mailbox.sc.edu

A. Hartstein

J. J. Wainer

A. B. Fowler

Follow this and additional works at: https://scholarcommons.sc.edu/phys_facpub

Part of the Physics Commons

Publication Info

Published in Physical Review Letters, ed. Gene D. Sprouse, Volume 54, Issue 14, 1985, pages 1577-1580.

Webb, R. A., Hartstein, A., Wainer, J. J., \& Fowler, A. B. (1985). Origin of the peaked structure in the conductance of one-dimensional silicon accumulation layers. Physical Review Letters, 54(14), 1577-1580. DOI: 10.1103/PhysRevLett.54.1577

(C) Physical Review Letters, 1985, American Physical Society

This Article is brought to you by the Physics and Astronomy, Department of at Scholar Commons. It has been accepted for inclusion in Faculty Publications by an authorized administrator of Scholar Commons. For more information, please contact digres@mailbox.sc.edu. 


\title{
Origin of the Peaked Structure in the Conductance of One-Dimensional Silicon Accumulation Layers
}

\author{
R. A. Webb, A. Hartstein, J. J. Wainer, and A. B. Fowler \\ IBM Thomas J. Watson Research Center, Yorktown Heights, New York 10598 \\ (Received 6 February 1985)
}

\begin{abstract}
We have made extensive studies of the temperature, gate voltage, and electric field dependences of the conductance peaks in small silicon inversion layers in order to distinguish between resonanttunneling models and a hopping model. We find that many of the peaks are consistent only with a hopping model, whereas some could be consistent with an early resonant-tunneling model. None of our structure is consistent with resonant tunneling if the recent formulation of Stone and Lee is correct.
\end{abstract}

PACS numbers: $71.55 . \mathrm{Jv}, 73.40 . \mathrm{Qv}$

An interesting and puzzling feature of the lowtemperature conductance observed in ultranarrowchannel silicon metal-oxide-semiconductor field-effect transistors (MOSFET's) is the large variation in conductance (as much as 2 orders of magnitude) when the electron density is changed by less than $0.05 \%$. This structure was unmistakable in the first studies ever made on these ultranarrow one-dimensional (1D) samples. $^{1-3}$ It was also present, but not as dramatically, in the experiments of other groups ${ }^{4-6}$ on different but related samples. Weaker structure was reported ${ }^{7}$ as long ago as 1965 in wide samples. In our previous work ${ }^{1-3}$ we characterized the gate voltage and temperature dependences of both the conductance maxima and minima and offered the suggestion that the explanation for these peaks might be found in statistical fluctuations in the density of states arising from the extremely small sample size.

Recently, two alternative suggestions for the origin of these peaks have been made. Azbel and coworkers ${ }^{8-10}$ suggested that the observed structure is due to resonant tunneling of electrons through the sample. Lee ${ }^{11}$ has considered the role of statistical fluctuations in small hopping systems where only one or two hops dominate the conductance. When parameters estimated for our sample are used in the model, the structure arises because below $V_{G} \approx 5 \mathrm{~V}$ there are fewer than 50 states within several $k_{\mathrm{B}} T$ of the Fermi energy at $100 \mathrm{mK}$. This extreme limit has the appealing feature that it allows for much larger fluctuations in the conductance than a simple density-of-states argument. The purpose of this Letter is to report experimental work which bears directly on the explanation of the origin of the peaked structure observed in the experiments. We find that certain peaks have features which are only consistent with the Lee model, and the remainder are in qualitative agreement with the models of Lee ${ }^{11}$ or Azbel and co-workers. ${ }^{8-10}$

The samples used in these experiments are pinched accumulation-layer Si MOSFET's, ${ }^{1-3}$ made on $10-\Omega$ cm $n$-type substrates. The source and drain contacts are $n$-type diffusions and the control electrodes are $p$ - type diffusions. The gate oxide is $30 \mathrm{~nm}$ thick, and the channel length is $10 \mu \mathrm{m}$. In the $1 \mathrm{D}$ regime we believe that the channel width is less than $30 \mathrm{~nm} .^{1,2}$

We have made conductance measurements using an ac lock-in technique at $50 \mathrm{~Hz}$ and a source-drain voltage of $2 \mu \mathrm{V}$. The control voltage applied to the $p^{+}$ regions was fixed at $0 \mathrm{~V}$. The sample was placed inside the mixing chamber of a dilution refrigerator, and measurements were made in the temperature range 23 $\mathrm{mK} \leqslant T \leqslant 1 \mathrm{~K}$. Data were obtained over the range of gate voltages $\left(3 \mathrm{~V} \leqslant V_{G} \leqslant 5 \mathrm{~V}\right)$ where, at temperatures above $200 \mathrm{mK}$, our sample shows 1D hopping conductance behavior. In this experiment we concentrated on narrow ranges of gate voltage which at low temperature contained individual, well-separated conductance peaks.

Figure 1 displays the conductance on a logarithm

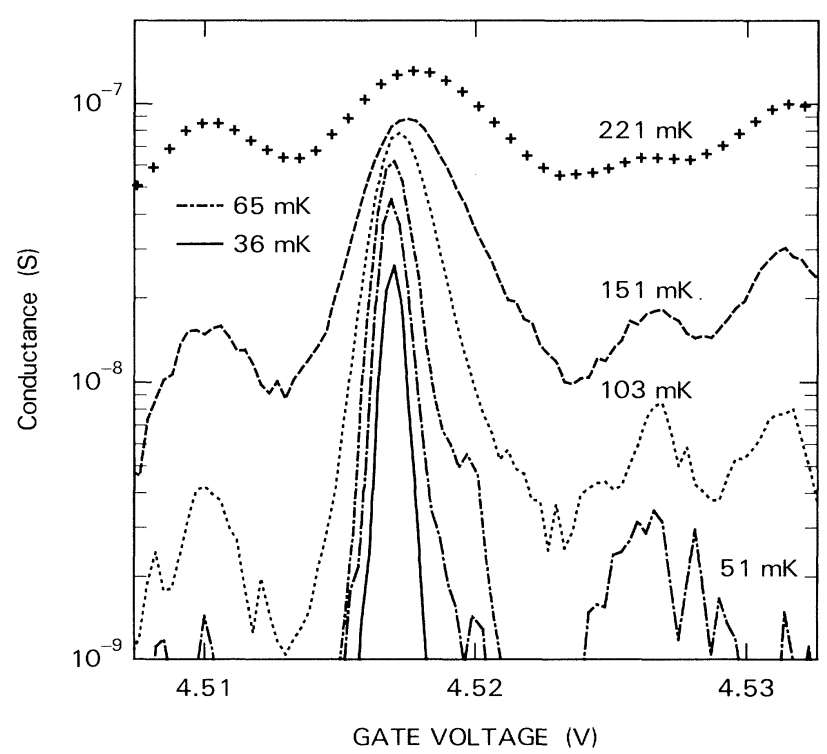

FIG. 1. Conductance as a function of gate voltage on an expanded gate voltage scale for selected temperatures. Only the large peak is displayed for the $65-$ and $36-\mathrm{mK}$ curves. 
scale as a function of gate voltage for one of the narrow regions studied at several representative temperatures. It is clear that over a reasonable range of temperature the peaks do not overlap, with significant overlap only setting in above $120 \mathrm{mK}$. The sides of the peaks vary linearly with gate voltage in these semilog plots (except very close to the top of the peak). The energy dependence of the conductance can be extracted from the gate voltage dependence. We can convert the gate voltage scale to an energy scale by using the gate capacitance, $1.2 \times 10^{-7} \mathrm{~F} / \mathrm{cm}^{2}$, and assuming that the 2D band density of $1 \mathrm{D}$ conduction states, $1.6 \times 10^{14} \mathrm{eV}^{-1} \mathrm{~cm}^{-2}$, is applicable on the average. The magnitude of the slopes are approximately $1 / k_{\mathrm{B}} T$ and in general slightly different on both sides of the peak. It can also be seen that for this gate voltage range the peak positions show a small but systematic shift with temperature.

Figure 2 displays the temperature dependence of the four largest peaks in Fig. 1. Above $200 \mathrm{mK}$, where the peak overlap is strong, the data for all the peaks are fitted well by the form

$$
\sigma=\sigma_{0} \exp \left[-\left(T_{0} / T\right)^{1 / 2}\right],
$$

which is characteristic of variable-range hopping in one dimension ${ }^{1-3}$ with $T_{0}$ and $\sigma_{0}$ as constants. For most of the peaks, the temperature region below $200 \mathrm{mK}$ can be fitted equally well with either a $T^{-1}$ or a $T^{-1 / 2}$ exponential temperature dependence. The data displayed by the open circles in Fig. 2 illustrate one example of a

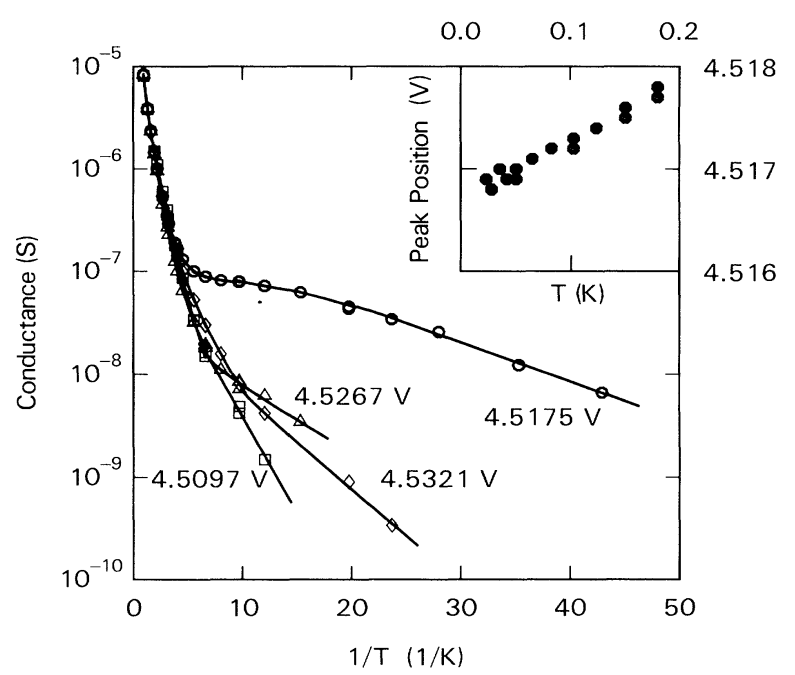

FIG. 2. Temperature dependence of four conductance peaks shown in Fig. 1 at $V_{G}=4.5097,4.5175,4.5267$, and $4.5321 \mathrm{~V}$ with activation energies $36,7.2,13$, and $18 \mu \mathrm{eV}$, respectively. Solid lines are smooth curves through the data. The inset shows the temperature dependence of the peak position for the peak at $V_{G}=4.5175 \mathrm{~V}$ in the temperature regime where the peak is isolated from its neighboring peaks. peak which deviates markedly from this behavior. The data are fitted by $\ln \sigma \propto 1 / T$ at low temperatures but flatten off at temperatures where $k_{\mathrm{B}} T$ is approaching the activation energy $(7.2 \mu \mathrm{eV})$. Of 120 peaks which we have studied in detail, five have the same qualitative behavior as this peak. It is worthwhile to note that of the remaining peaks about $10 \%$ are better fitted by a $1 / T$ dependence below $200 \mathrm{mK}$. In the rest of the cases the activation energy is too large and the temperature range too small to distinguish clearly between a $1 / T$ dependence and a $T^{-1 / 2}$ dependence. None are statistically better fitted by a $T^{-1 / 2}$ dependence in this low-temperature regime. All peak conductances were observed to increase with temperature above $23 \mathrm{mK}$.

One of the first theories put forward to explain the existence of the peaked structure is the resonanttunneling theory. The $T=0$ theory $^{8}$ demonstrates the existence of large, reproducible, sample-dependent structure in the conductance as a function of Fermi energy. The basic idea is that at special values of the Fermi energy constructive interference in the electronic wave functions can be expected, and this interference leads to a large increase in the transmission probability. Recently it has been argued by Azbel and coworkers 9,10 that when both thermal population effects and inelastic scattering are taken into account, the temperature dependence of the peaks should be

$$
\sigma=\sigma_{0} \exp \left[-\left(T_{0} / \eta T\right)^{1 / 2}\right],
$$

where $1 \leqslant \eta \leqslant 4$ and $\eta$ is a constant for any one peak, with 4 corresponding to unity transmission probability. As mentioned above, none of our low-temperature data can be best described by this equation. At high temperature the peaks merge and the average behavior is described by the normal variable-range-hopping conductance [Eq. (1)]. Stone and Lee ${ }^{12}$ have recently questioned the derivation of Eq. (2) and pointed out that the effect of inelastic scattering (finite $T$ ) should be to broaden the conductance peaks in such a way as to keep the integrated intensity constant. Thus the amplitude of the peaks should decrease with increasing temperature according to their derivation.

The inset to Fig. 2 shows the temperature dependence of the gate voltage position where we find maximum conductance for the $V_{G}=4.5175 \mathrm{~V}$ data. The peak position shifts linearly with temperature in the region where the peaks do not overlap. We have sufficiently accurate data to follow the peak shifts for six peaks: Two shift to positive gate voltage, two shift to negative gate voltage, and the remaining two do not shift. The size of the effect corresponds to a change in electron density of about $0.03 \%$ and depends on the particular peak being studied. We note that only two of these six peaks clearly demonstrate similar behavior as shown in Fig. 2 for the $4.5175-\mathrm{V}$ data. The resonant-tunneling model ${ }^{9,10}$ predicts that the peak po- 
sition should not shift with temperature, which is clearly inconsistent with our detailed observations.

The other theory which has been proposed involves a hopping model of the conduction in sparse systems where statistical fluctuations are important. Lee ${ }^{11}$ has shown that in a $1 \mathrm{D}$ hopping system with parameters comparable to our samples, fluctuations not only in the energy of each state but in the spatial location of the states can indeed produce structure as large as our observed behavior. He has also shown that the percolation problem reduces to only one or two critical hops, and that when one averages only over a relatively small number of peaks, the variable-range-hopping formula [Eq. (1)] is reproduced.

When a single critical hop dominates the current, the conductance in the linearized approximation with Boltzmann statistics is ${ }^{11}$

$$
\sigma=\sigma_{i j} \exp \left[-2 \alpha_{i j} r_{i j}-\left(1 / 2 k_{\mathrm{B}} T\right)\left(\left|E_{i}-\mu\right|+\left|E_{j}-\mu\right|+\left|E_{i}-E_{j}\right|\right)\right]
$$

where $1 / \alpha$ is the extent of the localized wave functions, $r_{i j}$ is the separation between hopping sites, $E_{i}$ and $E_{j}$ are the site energies, $\mu$ is the chemical potential, and $\sigma_{i j}$ is the appropriate prefactor for this process. This shows that a peak at low temperature will be simply activated, and that off of the peak the slope of the curves in Fig. 1 should correspond to $1 / k_{\mathrm{B}} T$ and be the same for both sides of the peak. Equation (3) predicts that the conduction is flat between $E_{i}$ and $E_{j}$ (finite width at zero temperature) and that the peak position is independent of temperature.

However, when two hops are taken in series, the situation qualitatively changes. If we describe each hop by Eq. (3), form the series combination, and solve for the peak position, we obtain

$$
E_{p}=\left(E_{i}+E_{1}\right) / 2+k_{\mathrm{B}} T\left(\alpha_{k l} r_{k l}-\alpha_{i j} r_{i j}\right)+\left(k_{\mathrm{B}} T / 2\right) \ln \left(\sigma_{i j} / \sigma_{k l}\right),
$$

where $E$ is the energy at one of the four sites $i, j, k$, and $l$ involved in the hopping process, $E_{i}$ and $E_{l}$ are the extremal energies, and $1 / \alpha$ is the corresponding wave-function decay constant. This type of peak does not have a flat top and shifts linearly in temperature with a magnitude and sign given by the asymmetry of the hopping sites involved.

If a single peak persists to high enough temperature without overlap from neighboring peaks, the Boltzmann approximation used in Eq. (3) is no longer valid. Equation (3) then generalizes to

$$
\sigma=\left[\sigma_{i j} \exp \left(-2 \alpha_{i j} r_{i j}\right)\right]\left\{F\left(E_{i}\right)\left[1-F\left(E_{j}\right)\right] N\left(E_{j}-E_{i}\right)-F\left(E_{j}\right)\left[1-F\left(E_{i}\right)\right]\left[1+N\left(E_{j}-E_{i}\right)\right]\right\},
$$

where $F$ is the Fermi function and $N$ is the Bose function. If a voltage is applied, $E_{j}-E_{i}$ must be modified. ${ }^{11}$ However, when all of the resistance is between sites $i$ and $j$, the Fermi functions are not modified by the applied voltage but take their equilibrium values. A similar but more complicated equation can be written for the case where two hops are in series. It is clear that deviations from a simple activated behavior can be expected. The data for the peak at $4.5175 \mathrm{~V}$ shown in Fig. 2 demonstrate the deviations from simple activated behavior contained in Eq. (5). Five of the peaks show deviations from simple activated behavior which can be described by the two-hop version of Eq. (5). No flat-topped peaks characteristic of one-hop-controlled processes were observed. Closer inspection of Eq. (4) reveals that the temperature dependence of the peak position can be either positive or negative and agrees with our observations that some peaks shift toward higher gate voltages while others shift toward lower voltages. Thus some of the peaks have properties which are consistent with the hopping model and clearly not consistent with the resonanttunneling model.

The resonant-tunneling model ${ }^{9,10}$ predicts that each peak corresponds to a resonant transmission state and that off of resonance it is believed that one should see a thermal activation into the resonant state. Thus the slopes of the conductance curves in Fig. 1, when the gate voltage axis is converted to an energy scale, should be $1 / k_{\mathrm{B}} T$. In fact, both models predict that the slopes of these curves as a function of $\mu$ should be $1 / k_{\mathrm{B}} T$, but clearly for different reasons. Experimentally, we can best describe our slopes by $\gamma / k_{\mathrm{B}} T$, where $0.3 \leqslant \gamma \leqslant 3$ and $\gamma$ is weakly temperature dependent. This is inconsistent with both models. It is possible that our assumed relationship between gate voltage and carrier concentration, which invokes a smoothly varying density of states, might break down in our narrow sample leading to the above result, but this is speculative. Including Coulomb interactions in the hopping model would lead to a small shift in the energy levels as the carrier density increased. In turn, the shift would modify the value of $\gamma$ and cause the slopes on either side of a conductance peak to differ. ${ }^{13}$

If one considers a hopping model in which only one or two hops control the conductance, it becomes clear that most of the source-drain voltage drop must appear across these hopping sites. Contained within Eq. (5) and discussed by Lee ${ }^{11}$ is the fact that the conductance can be asymmetric in the applied voltage about $V=0$. Therefore, one must anticipate nonlinear $I-V$ characteristics, rectifying behavior, and a generation of second-harmonic signals associated with individual peaks at low temperature in the hopping model. To a first approximation, we do not expect rectifying 


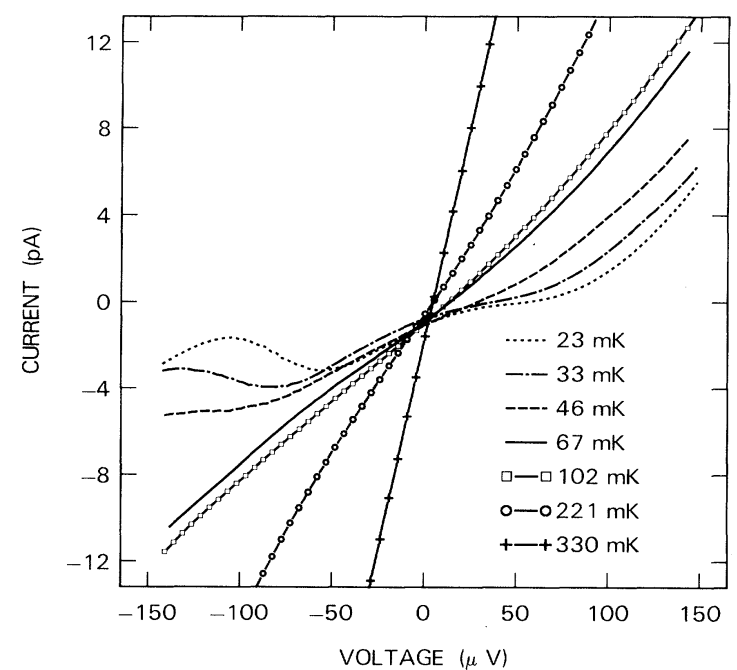

FIG. 3. The nonlinear $I-V$ curves observed for an isolated peak $\left(V_{G}=4.5175 \mathrm{~V}\right)$ at selected low temperatures.

behavior at a peak for small electric fields in a resonant-tunneling model.

Figure 3 is an example of the $I-V$ characteristics of the peak at $V_{G}=4.5175 \mathrm{~V}$ for several temperatures. One can clearly see that at low temperatures the curve is both nonlinear and highly asymmetric or rectifying. In addition, a second-harmonic signal is observed to grow from 30 to $150 \mu \mathrm{V}$ and then decrease in amplitude above $150 \mu \mathrm{V}$. Similar behavior to that displayed in Fig. 3 has been observed for many of our peaks. However, about $25 \%$ of our peaks show no measurable asymmetry about $V=0$ and for others the effect is not nearly as pronounced. This is not the usual $\sigma E^{2}$ heating effect frequently observed in $I-V$ curves at low temperatures. For our samples at low temperature, this conventional heating effect occurs at source-drain voltages in excess of $200 \mu \mathrm{V}$. The $I-V$ curves shown in Fig. 3 at the two lowest temperatures show a negative differential resistance region at negative $V_{\mathrm{SD}}$. We have not observed this for any of our other peaks but point out that in a hopping model one should expect modified behavior in the $I-V$ characteristics when the source-drain voltage becomes comparable to the ener- gy difference between hopping sites.

In summary, a fluctuation model of hopping conductivity with several series hops explains most of our detailed observations. These include the rectifying behavior of many of our peaks, the simply activated low-temperature behavior distinguishable for some of our peaks, and the temperature-dependent peak position of several of the sharpest peaks. The resonanttunneling model as formulated by Azbel and coworkers 9,10 is consistent only with those peaks for which the precise form of the temperature dependence at the very lowest temperatures cannot be clearly ascertained. And if the more recent formulation of Stone and Lee ${ }^{12}$ is correct, then none of the observed peaks can be due to resonant tunneling.

We wish to thank M. Ya. Azbel, P. A. Lee, A. D. Stone, G. L. Timp, and S. Washburn for helpful discussions.

${ }^{1}$ A. B. Fowler, A. Hartstein, and R. A. Webb, Phys. Rev. Lett. 48, 196 (1982).

${ }^{2}$ A. B. Fowler, A. Hartstein, and R. A. Webb, Physica (Utrecht) $117 \& 118 B, 661$ (1983).

${ }^{3}$ A. Hartstein, R. A. Webb, A. B. Fowler, and J. J. Wainer, Surf. Sci. 142, 1 (1984).

${ }^{4}$ W. J. Skocpol, L. D. Jackel, E. L. Hu, R. E. Howard, and L. A. Fetter, Phys. Rev. Lett. 49, 951 (1982).

${ }^{5}$ R. G. Wheeler, K. K. Choi, A. Goel, R. Wisnieff, and D. E. Prober, Phys. Rev. Lett. 49, 1674 (1982).

${ }^{6}$ R. F. Kwasnick, M. A. Kastner, J. Melngailis, and P. A. Lee, Phys. Rev. Lett. 52, 224 (1984).

${ }^{7}$ W. E. Howard and F. F. Fang, Solid-State Electron. 8, 82 (1965).

${ }^{8}$ M. Ya. Azbel and P. Soven, Phys. Rev. B 27, 831 (1983).

${ }^{9}$ M. Ya. Azbel, A. Hartstein, and D. P. DiVicenzo, Phys. Rev. Lett. 52, 1641 (1984).

${ }_{10}$ M. Ya. Azbel and D. P. DiVincenzo, Phys. Rev. B 30, 6877 (1984).

11P. A. Lee, Phys. Rev. Lett. 53, 2042 (1984).

${ }^{12}$ A. D. Stone and P. A. Lee, Phys. Rev. Lett. 54, 1196 (1985).

13P. A. Lee, private communication. 